\title{
Drugs Prescribed by Dentists in Fars Province, Iran
}

\author{
Mohammad Mehdi Fani ${ }^{1}$; Mustafa Ghaeminia ${ }^{2}$; Amin Farjood ${ }^{3, *}$ \\ ${ }^{1}$ Department of Oral Medicine, School of Dentistry, Shiraz University of Medical Sciences, Shiraz, IR Iran \\ ${ }^{2}$ Department of Food and Drug, Shiraz University of Medical Sciences, Shiraz, IR Iran \\ ${ }^{3}$ Student Research Committee, School of Dentistry, Shiraz University of Medical Sciences, Shiraz, IR Iran \\ *Corresponding author: Amin Farjood, Student Research Committee, School of Dentistry, Shiraz University of Medical Sciences, Shiraz, IR Iran. Tel: +98-9171052084, E-mail:amin31f@ \\ yahoo.com \\ Received: June 29, 2013; Accepted: July 4, 2013
}

\begin{abstract}
Background: Medication is one of the supplementary steps in dental treatment. Dentists prescribe medicine in order to avoid postsurgery complications. To the best knowledge of the researchers, no thorough study has been carried out to review the prescribed drugs in Fars province, Iran.

Objectives: The current study aimed to determine the current status of the issue and compare it with global standards.

Materials and Methods: In this cross sectional study, 1120528 prescriptions prescribed by the dentists of Fars province from 2006 to 2011 were reviewed in terms of the number of items, the types of drugs and the most commonly prescribed drugs.

Results: The average number of drugs in every prescription in Fars province, Iran was $2.26 \pm 0.56$. About $82 \%$ of the prescriptions included at least one antibiotic and $21 \%$ included at least one corticosteroid. Amoxicillin (23.71\%), metronidazole (10.6\%) and ibuprofen (7.5\%) were the most common prescribed drugs.

Conclusions: Compared to the developed countries, dentists of Fars acted properly regarding the average number of drugs found in every prescription. But in prescribing antibiotics, corticosteroids and injections they need further education.
\end{abstract}

Keywords:Dentists; Prescriptions; Antibacterial Agents; Adrenal Cortex Hormones

\section{Background}

Drug prescription is indispensible to medical procedures. Despite its crucial role in treating patients, there are still some prescriptions which do not follow the standards set by health authorities. That is to say, in spite of all efforts, irrational ways of prescription are still common all over the world (1). Wrong prescription, in addition to patients' financial loss, can cause adverse effects such as microbial resistance, toxicity, etc. $(2,3)$. In fact, in a conference held in Nairobi in 1985, an international effort for logical prescription started. After that, the World Health Organization (WHO) and other international organizations investigated this issue (4). The findings of the studies showed that drugs adverse effects were the fourth cause of mortality in developing countries (5).

Irrational prescription of drugs is common in many countries including developed countries (3). Different studies showed that average number of drugs in every prescription in many countries outnumbers the WHO standards (6). Furthermore, there is ample evidence testifying the limited knowledge of dentists in prescribing medicine in various countries (7-9). In this regard, the WHO has submitted some indices as the criteria of logical drug consumption, which are classified in three sections: patient care, prescription, and health facilities.

\section{Objectives}

According to the importance of logical prescription of medicine and due to limited studies on dentists' prescription patterns performed so far in only a few cities of Iran, such as Kerman (10), the present study was carried out to establish some indices such as the number of drugs in every prescription, application of drugs prescribed, and the frequency of different drug categories among the dentists of Fars province. It should be noted that the research performed in Kerman included only a limited number of prescriptions and the difference between general dentists and specialists was not considered.

\section{Materials and Methods}

In this cross sectional study, all prescriptions made from 2006 to 2011 were reviewed and related information was retrieved (census). All prescriptions covered by insurance companies made by dentists in Fars, were collected every month by rational use of drug committee and their information was extracted and recorded. The information was reviewed by the authors and related data were extracted. It is worth mentioning that only the medicines covered by insurance companies in Fars, by referring to rational use of drug committee, were reviewed in the current study. The average number of drugs in every 
Fani MM et al.

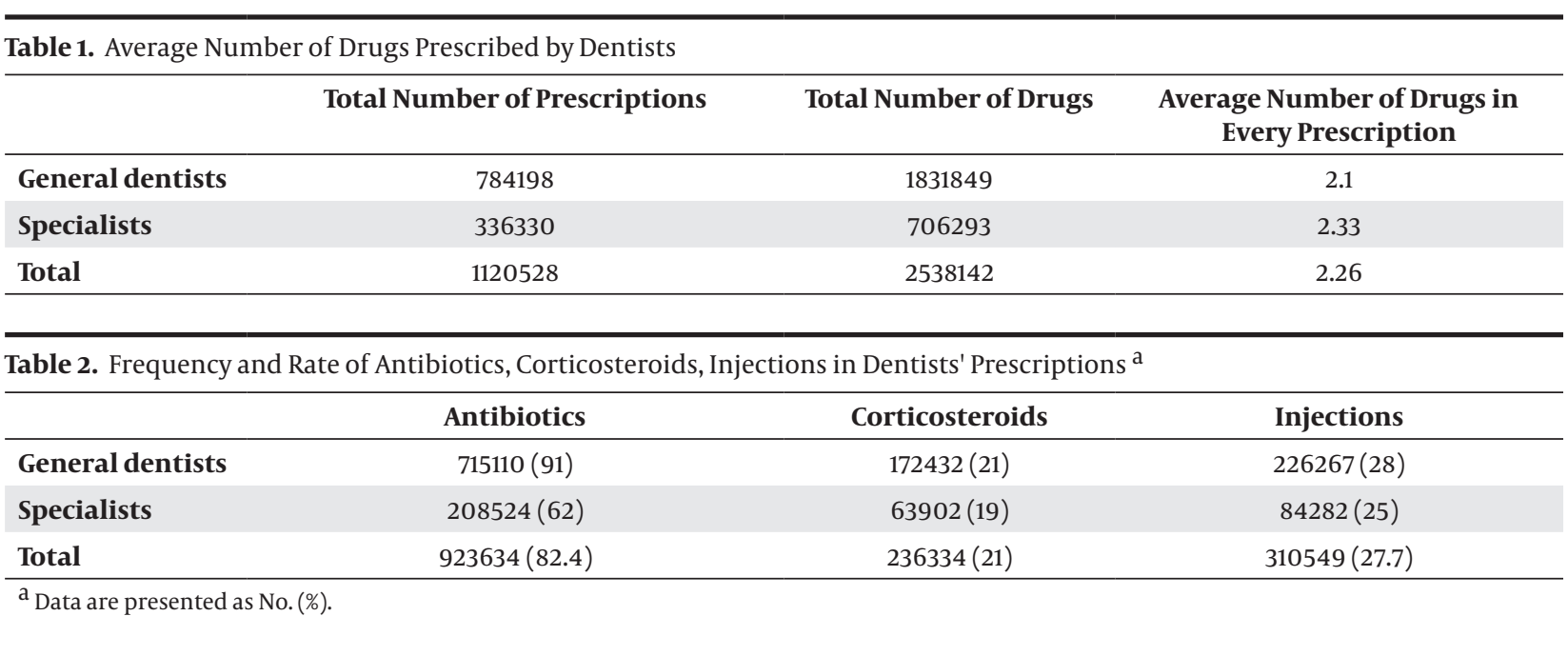

Table 3. The Type and Frequency of the Different Drugs Prescribed by Dentists ${ }^{\text {a }}$

\begin{tabular}{|c|c|c|c|c|c|c|c|c|}
\hline & Amoxicillin I & Metronidazole & Ibuprofen & dexamethasone & Acetaminophen & penicillin & Mefenamic acid & Total \\
\hline $\begin{array}{l}\text { General } \\
\text { dentists }\end{array}$ & $441979(24.1)$ & $236200(12.8)$ & $159416(8.7)$ & $149554(8.1)$ & $104675(5.7)$ & $107341(5.8)$ & $102792(5.6)$ & 1831849 \\
\hline Specialists & $160031(22.6)$ & $34890(4.9)$ & $32220(4.5)$ & $39650(5.6)$ & $49670(7)$ & $19230(2.7)$ & $13260(1.8)$ & 706293 \\
\hline
\end{tabular}

a Data are presented as No.(\%)

prescription, the rate of antibiotics, corticosteroids, and drugs for injection were calculated. Moreover, the most common drugs prescribed were also reported. Data were analyzed by SPSS v. 17.0, and then mean and standard deviation were calculated.

\section{Results}

Among 1120528 reviewed prescriptions, 784198 were prescribed by general dentists and 336330 by specialists. The average number of drugs in every prescription in Fars province was $2.26 \pm 0.56$ (Table 1). In total, $82.42 \%$ of prescriptions ordered by the dentists of Fars included at least one antibiotic. Moreover, in $21 \%$ of all prescriptions, there was at least one corticosteroid. It was also noticed that, compared with specialists, general dentists prescribed these drugs more frequently (Table 2). In addition to antibiotics, corticosteroids, injections, the type and frequency of different drugs prescribed were reviewed. Table 3 shows the type and frequency of the different drugs prescribed by the dentists of Fars province. Amoxicillin was the most common drug prescribed (23.71\%), followed by metronidazole and ibuprofen.

\section{Discussion}

It is obvious that prescribing excessive drugs not only increases the possibility of adverse drug reactions, but also causes some financial losses on behalf of both the patient and the society. The average number of drugs prescribed by dentists of Fars was 2.26, compared to those of Kerman (2.03), as reported in a similar study (10). Concerning the number of drugs in every prescription, there was no sig- nificant difference between dentists of Fars and Kerman Provinces, Iran. To the best knowledge of the researchers, there were no reports on the number of drugs in the dentists' prescriptions in other areas; but considering the average number of drugs prescribed by general practitioners in the developed countries (1.3 to 2.2) (6), the results of the current study indicated a relatively good performance by the dentists of Fars in this regard. The performance of general dentists of Fars was similar to those of specialists in number of drugs prescribed.

Since health providers, nowadays, are and more conscious about bacterial infections, they more frequently recommend antibiotics. However, they should be quite aware of the side effects of antibiotics while prescribing them (11).

The rate of antibiotics prescribed by dentists of Fars (82.4\%) was almost the same as those of Kerman (82.2\%) (10), which was in contrast to the rate of antibiotics prescribed by the dentists in Norway and New York, 65\%, and $52.2 \%$, respectively $(12,13)$. Concerning antibiotics prescription, the performance of the specialists in Fars was much better than those of general dentists. Taking different kinds of antibiotics prescribed, while amoxicillin, metronidazole and cephalexin were the most common antibiotics prescribed by dentists in Fars, the most commonly prescribed antibiotics by dentists in Belgium were amoxicillin, clindamycin and azithromycin (14) and the most common antibiotics in prescriptions of dentists in New York was penicillin, followed by clindamycin and erythromycin (15).

It is noteworthy that metronidazole was not among the five most common antibiotics prescribed in any 
areas except Fars. Although metronidazole only affects anaerobe bacteria, its efficacy as an antibiotic in management of odontogenic infections has been confirmed in different studies (16).

Despite the fact that indications for antibiotic therapy have increased these days, it should not be considered a treatment for all conditions, and a substitution for procedures such as incision and drainage. Moreover, when prescribing antibiotics, adverse drug reactions should always be taken into account (11).

Ibuprofen and acetaminophen were the most commonly prescribed analgesics in prescriptions of the Fars dentists. an article conducted in 2010 revealed that acetaminophen and NSAIDs were prescribed more than any other analgesics (17). Compared with Kerman (1.4\%), the rate of corticosteroids prescribed by the dentists of Fars was higher (21\%). Various studies have emphasized that in dentistry the use of corticosteroids should be limited to specific conditions. Although the effects of corticosteroids, such as reducing post-treatment pain and swelling, is well known, they should be used with care and be administered locally whenever possible, due to their side effects they cause (18-20). Studying the patterns of drug prescription of dentists in New York, the rate of drug injection was only 1.5\%. This amount was 20 times higher among dentists of Fars, indicating the poor performance of Fars dentists (12). In summary, comparing dentists of Fars with dentists and general practitioners in other areas with regard to the average number of drugs found in every prescription, it can be concluded that the performance of Fars dentists in this regard is appropriate.

But the rate of antibiotics, corticosteroids and drug injection prescribed by the dentists of Fars was very high. It is noteworthy that concerning the prescription of the three above mentioned drugs the performance of specialist dentists was better than that of the general dentists. There are many reasons underlying the poor performance of Fars dentists which can be the subject of further studies. For instance, the education program assigned for the general dentistry can be scrutinized. That is, it might be necessary to revise the curriculum of general dentistry, placing more emphasis on the importance of drug prescription.

\section{Acknowledgements}

The authors acknowledge their gratitude to the Vicechancellery of Shiraz University of Medical Sciences for supporting this research (Grant \#5898). This article is based on the thesis by Dr. Amin Farjood. The authors also thank Dr. Mehrdad Vosughi, a member of Dental Research and Development Center of Dentistry School, for the statistical analysis and Dr. Ehya Amalsaleh for English editing.

\section{References}

1. Keers RN, Williams SD, Cooke J, Ashcroft DM. Causes of medication administration errors in hospitals: a systematic review of quantitative and qualitative evidence. Drug Saf. 2013;36(11):1045-67.

2. Classen DC, Pestotnik SL, Evans RS, Lloyd JF, Burke JP. Adverse drug events in hospitalized patients. Excess length of stay, extra costs, and attributable mortality. JAMA. 1997;277(4):301-6.

3. Laing R, Hogerzeil H, Ross-Degnan D. Ten recommendations to improve use of medicines in developing countries. Health Policy Plan. 2001;16(1):13-20.

4. Riedl MA, Casillas AM. Adverse drug reactions: types and treatment options. Am Fam Physician. 2003;68(9):1781-90.

5. Ponte ML, Ragusa M, Armenteros C, Wachs A. [Importance of pharmacovigilance in current medical practice]. Medicina (B $A i$ res). 2013;73(1):35-8.

6. Hogerzeil HV, Walker GJ, Sallami AO, Fernando G. Impact of an essential drugs programme on availability and rational use of drugs. Lancet. 1989;1(8630):141-2.

7. Mendonca JM, Lyra DP, Jr, Rabelo JS, Siqueira JS, Balisa-Rocha BJ, Gimenes FR, et al. Analysis and detection of dental prescribing errors at primary health care units in Brazil. Pharm World Sci. 2010;32(1):30-5.

8. Cherry WR, Lee JY, Shugars DA, White RP, Jr, Vann WF, Jr. Antibiotic use for treating dental infections in children: a survey of dentists' prescribing practices. J Am Dent Assoc. 2012;143(1):31-8.

9. Guzman Alvarez R, Campos Sepulveda AE, Martinez Gonzalez AA. Knowledge about local anesthetics in odontology students. Proc West Pharmacol Soc. 2009;52:118-9.

10. Sepehri G, Dadolahi Y. Characterization of drug prescribing practices among dentists in Kerman province, 2001. J Dent Sch. 2006;24(1):7.

11. Seymour RA. Antibiotics in dentistry--an update. Dent Update. 2013;40(4):319-22.

12. Ciancio S, Reynard A, Zielezny M, Mather M. A survey of drug prescribing practices of dentists. NYState Dent J.1989;55(1):29-31.

13. Demirbas F, Gjermo PE, Preus HR. Antibiotic prescribing practices among Norwegian dentists. Acta Odontol Scand. 2006;64(6):355-9.

14. Mainjot A, D'Hoore W, Vanheusden A, Van Nieuwenhuysen JP Antibiotic prescribing in dental practice in Belgium. Int Endod J. 2009;42(12):1112-7.

15. Yingling NM, Byrne BE, Hartwell GR. Antibiotic use by members of the American Association of Endodontists in the year 2000: report of a national survey. J Endod. 2002;28(5):396-404.

16. Peterson LJ. Contemporary oral and maxillofacial surgery. 4 th ed. St. Louis: Mosby; 2003.

17. Donaldson M, Goodchild JH. Appropriate analgesic prescribing for the general dentist. Gen Dent. 2010;58(4):291-7. quiz 298-9.

18. Rogers MJ, Johnson BR, Remeikis NA, BeGole EA. Comparison of effect of intracanal use of ketorolac tromethamine and dexamethasone with oral ibuprofen on post treatment endodontic pain. JEndod.1999;25(5):381-4.

19. Negm MM. Intracanal use of a corticosteroid-antibiotic compound for the management of posttreatment endodontic pain. Oral Surg Oral Med Oral Pathol Oral Radiol Endod. 2001;92(4):435-9.

20. Hargreaves K, Abbott PV. Drugs for pain management in dentistry. Aust Dent J. 2005;50(4 Suppl 2):S14-22. 